group. BMD of the forearm was measured by DXA using DTX200 and QUS parameters by DTU-ONE. BMD of lumbar spine and the hip were measured by DXA using ECLIPSE densitometer. Disease activity was assessed by measuring serum levels of proinflammatory cytokines (IL-1a, IL-6, TNF-alpha, GM-CSF) and acute phase markers (ESR, CRP, AGP, AGP-RC, ACT).

Results In RA pts on GSC therapy the disease activity was lower and also bone turnover was decreased. Moreover, in SLE pts with the highest dosage of GCS and the longest duration of the therapy the decrease in bone mass and the bone loss was the lowest.

Conclusion Our results show questionable correlation between bone mass and bone loss in patients with chronic connective tissue diseases on long-term and low-dose corticosteroids therapy. Nevertheless, in long-term GCS treatment of RA pts and SLE pts bone decomposition is probably higher than anti-inflammatory effect.

\section{AB0191 PREVALENCE OF OSTEOPOROSIS AMONG FEMALE SYSTEMIC LUPUS ERYTHEMATOSUS PATIENTS}

O Di Munno, M Mazzantini, M Mosca, S Frigelli, A Delle Sedie, R Neri, S Bombardieri. Rheumatology and Clinical Immunology Unit, Department of Internal Medicine, University of Pisa, Pisa, Italy

\subsection{6/annrheumdis-2001.630}

Background It has been reported that bone mineral density (BMD) is significantly reduced in premenopausal SLE patients as compared to controls and that $12-25 \%$ of such patients are considered to have osteoporosis (OP). Furthermore, SLE patients have a 5 -fold probability of sustaining a fracture as compared to the normal population. Long term glucocorticoids (GC) and immunosoppressive drugs are likely to play a major role in the pathogenesis of bone loss.

Objectives Aim of the present study was to assess BMD and OP prevalence among ambulatory female SLE patients with respect to a normal population.

Methods Thirty-two pre- (mean age $36 \pm 9$ years) and 14 postmenopausal (mean age $57 \pm 8$ ) patients have been enrolled so far. Exclusion criteria were: any other disease known to affect bone mass, renal insufficiency (serum cretinine $>2 \mathrm{mg} / \mathrm{dl}$ for $>$ 6 months), any treatment for $>1$ month for the prevention and treatment of bone loss, excluded calcium and vitamin D supplementation. Lumbar and femoral BMD were measured by dual $\mathrm{x}$ ray absorptiometry (DXA). All patients were receiving GC at the time of the study. Variables included were: body mass index, disease duration, activity (ECLAM) and damage (SLICC), duration of GC treatment, cumulative dose of GC (either by pulse therapy and daily oral dose), and use of the immunosoppressive agent cyclophosphamide.

Results In premenopausal patients mean lumbar BMD was 0.96 $\pm 0.13 \mathrm{~g} / \mathrm{cm}^{2}$ (mean T-score $-0.8 \pm 1.2$ ), and femoral BMD was $0.87 \pm 0.19 \mathrm{~g} / \mathrm{cm}^{2}$ (T-score $-0.8 \pm 1.1$ ); in postmenopausal patients lumbar BMD was $0.85 \pm 0.13 \mathrm{~g} / \mathrm{cm}^{2}$ (T-score $-1.8 \pm$ 1.2) and femoral BMD $0.84 \pm 0.11$ (T-score $-1.2 \pm 0.9$ ). OP, defined as a T-score below 2.5 SD compared to a reference population of young healthy women in at least one region of measurement, was detected in $4(12.5 \%)$ premenopausal and in 3 (21.4\%) postmenopausal patients. Comparison to the normal population and the correlations between BMD and the variables included will be evaluated only after the completion of the recruitment.
Conclusion These preliminary results show in our SLE patients a prevalence of OP which is similar to that reported in other series. The role of the variables included in the analysis will help understand the pathogenesis of bone loss.

\section{AB0192 INFLUENCE OF EXERCISE ON BONE MINERAL DENSITY}

SC Brankovic, NZ Pilipovic, P Vukojevic. Physical Therapy, Institute of Rheumatology, Belgrade, Yugoslavia

10.1136/annrheumdis-2001.631

Background Parameters of the ideal exercise programs, such as type, frequency, intensity and duration of activity are unknown. When women with sedentary way of life $(<30 \mathrm{~min}$ of exercise per week) began a strenuous training program, bone mass increased significantly. Regular physical activity may help to maintain skeletal integrity throughout life and possibly reduce the long-term risk for fracture. Exercise intervention may provide modest increases in bone mass, but after one year period of detraining, BMD had returned to baseline, indicating that exercise must be sustained for bone mineral improvements to persist. Objectives The aim of study was to assess the effects of our exercise program on bone mineral density in the prevention and treatment of osteoporosis.

Methods We developed a exercise program in Institute of Rheumatology and the patients with low bone mineral density (BMD) exercised 3 times a week (for 4 weeks). After that period patients continued regularly with exercise at home. BMD was measured by dual x-ray absorptiometry (Lunar DXA system).

Results Group of 24 pts who only exercised without taking any drugs for osteoporosis, mean age 57,38 (46-73) years and mean BMD 0,915 $\pm 0,12 \mathrm{gr} / \mathrm{cm}^{2}$ with average T score $-2,40 \pm 0,98$. The control DXA was done after average period of 10,3 months. BMD was increased to mean $0,925 \pm 0,10 \mathrm{gr} / \mathrm{cm}^{2}$ $(1,5 \%)$, and $\mathrm{T}$ score to $-2,28 \pm 0,88(3,7 \%)$, (N. S.) Sixteen patients with BMD 9,23 $\pm 0,07 \mathrm{gr} / \mathrm{cm}^{2}$ did not continued with exercising, and after average 11,7 months BMD was decreased to $9,04 \pm 0,06 \mathrm{gr} / \mathrm{cm}^{2}(2,1 \%) ;(\mathrm{t}=2,21 ; \mathrm{p}<0,05)$

Conclusion Our result suggests that exercise have a positive effect on BMD even without medical therapy. It is necessary to continue with exercise at home because BMD decreased more than returned to baseline.

\section{OP0111 TREATMENT OF ESTABLISHED CORTICOID-INDUCED OSTEOPOROSIS BY 3-MONTHLY INTRAVENOUS IBANDRONATE INJECTIONS}

JD Ringe, A Dorst, H Faber. Medicine Klinik IV, Klinikum Leverkusen, University of Cologne, Germany

\subsection{6/annrheumdis-2001.632}

Background Long-term anti-inflammatory therapy with corticosteroids (CS) leads to decreased formation and increased resorption of bone, i.e. to corticoid-induced osteoporosis (CIO). Several recent studies with etidronate, alendronate and risedronate has proven the efficacy of oral bisphosphonate (BP) treatment to increase bone mineral density (BMD) and reduce fracture risk in these patients. Since chronically ill patients with CIO often take multiple oral medications, an injectable BP may improve compliance and therapeutic results.

Objectives 